\title{
THE STUDY OF THE STABILITY OF THE GEL FORMULA AND THE ABSORPTION IN THE HAIR OF BLUE PEA EXTRACT GEL AND CRC EXTRACT GEL
}

\section{(C) Chaipradit K. ${ }^{1}$, Priprem A. ${ }^{2}$, Nualkaew S. ${ }^{3}$, Preeprame S. ${ }^{2}$}

'Borabeu hospital, Amphur borabeu, Mahasarakam Thailand

${ }^{2}$ Faculty of pharmaceutical sciences, Khon Kaen University, Thailand

${ }^{3}$ Faculty of pharmaceutical sciences, Mahasarakam University, Thailand

The major objective of this study was the stability blue pea extract gel and CRC extract gel and the adsorption distance of the gel in the gray hair. Blue pea extract was prepared by extraction of the petal of blue pea (Clitoria ternatea L.) in hot water for 2 hours and evaporate to 1 gram per 1 milliliter concentration. Blue pea extract gel was formulated with $32 \% \mathrm{w} / \mathrm{v}$ blue pea extract, $2 \%$ hydroxypropylmethylcellulose, $30 \%$ of ethyl alcohol $0.5 \%$ Bronidox $0.17 \%$ triethanolamine and purify water to $100 \mathrm{ml}$. CRC extract was the combination of anthocyanin extract from rose and blue pea $(\mathrm{C}=$ Clitorea ternatea $\mathrm{L}$., $\mathrm{R}=$ Rosa hybrida $\mathrm{L}$., $\mathrm{C}=$ complex). The CRC extract was in patent process and was obtained from associated professor Aroonsri Priprem, faculty of pharmaceutical sciences, Khon Kaen University Khon Kaen Thailand. CRC extract gel was formulated with $0.1 \%$ CRC extract $2.5 \%$ hydroxypropylmethylcellulose, $60 \%$ ethyl alcohol, $0.5 \%$ Bronidox, $0.4 \%$ triethanolamine and purify water to $100 \mathrm{ml}$. The gels formulated were tested the stability by evaluated the color, $\mathrm{pH}$, viscosity and clarity after freeze and thaw condition for 3 cycles. The adsorption distances in the gray hair were evaluated by measuring distance of color absorption from out layer to inside of the hair within 5 minute after applying the gel to the hair. The percent of adsorption in the hair in $5 \mathrm{~min}-$ ute of blue pea extract gel, CRC extract gel, $0.1 \% \mathrm{w} / \mathrm{v}$ lawsone in ethanol and benchmark product containing $p$-Phenylenediamine (PPD) were calculated and com- paring the adsorptions character using microscope. The permanent of the staining were evaluated by apply the staining gel to the hair for 1 minute and leaved on 5 minutes, then measuring the adsorption distance in the hair, then washing the hair with baby shampoo and water for 1 minute, leave the hair dry for 5 minutes and observed the color and the absorption distance, repeated the washing step and observed the color and absorption change 4 times. After freeze and thaw condition the color of blue pea gel was not change, the color of CRC gel was change 2 color scale. The pH of Blue pea gel decrease $4.8 \%$ and $\mathrm{pH}$ of $\mathrm{CRC}$ gel increase $0.9 \%$. The viscosity of blue pea extract gel was increased $3.4 \%$ and viscosity of the CRC extracted gel was increased $1.6 \%$. The clarity of the gel was not change. The absorption distance of the CRC extract gel was more than Blue pea gel significantly. The CRC extract gel and the benchmark product contain PPD had none significantly absorption distance. The permanent of hair color after washing showed that the blue pea extracted gel could not remain hair color, the CRC extract gel and $1 \%$ lawsone solution remain hair color for 2 time of washing, the benchmark product contain PPD remain hair color for 4 time of washing. The further development of CRC extracted gel for hair staining should focus on improvement of the permanent staining property which could promoted this substances to be an alternative natural and non toxic hair staining in the market.

\section{EFFECT OF CRUDE EXTRACTS OF PHYLLANTHUS NIRURI L. TOWARDS PLATELET AGGREGATION EVALUATED IN 96-WELLS MICROPLATE READER}

\section{() Puspita N. A., Pye D.}

Centre for Biochemistry, Drug Design and Cancer Research, University of Salford, U. K. M45WT

Phyllanthus niruri L. is one of tropical plants that have been widely used in many countries as a traditional remedy for treating haemorrhagic diseases, particularly in the case of low platelet count and disturbance of platelet functions. However, literature discussing its pharmacological effects in platelet functions is very limited. The aim of this study is to explore the effect of crude Phyllantus niruri L. extracts towards inhibition of plate- let aggregation. The plant is extracted with maceration method using various solvents; hexane, chloroform, ethanol, methanol, and water. Adenosine 5'-diphosphate as an activating agent is used to induce platelet aggregation; the extent of inhibition was monitored in 96-wells microplate reader. The result (tab.) demonstrated that hexane, chloroform, ethanol, methanol, and water extracts of Phyllanthus niruri L. in concentration 
Table. Platelet Aggregation Inhibition

\begin{tabular}{|c|c|c|c|c|c|c|}
\hline & Adenosine & Water Extract & Hexane Extract & Chloroform Extract & Ethanol Extract & Methanol Extract \\
\hline Inhibition (\%) & $68.6 \pm 4.3$ & $72.7 \pm 3.3$ & $40.1 \pm 5.6$ & $44.7 \pm 2.7$ & $43.5 \pm 6.6$ & $68.8 \pm 6.7$ \\
\hline
\end{tabular}

of $250 \mathrm{mg} / \mathrm{ml}$ showed a potent inhibition effect toward platelet aggregation. Compared with other extracts, water extract exhibited the strongest inhibition as well as adenosine $(50 \mathrm{uM} / \mathrm{ml})$ as a positive control.
In Addition to this, methanol extracts showed relatively similar inhibition pattern with adenosine. Finally, this initial finding has provided the evidence of Phyllantus niruri L. action in platelet functions.

\title{
PRODUCTION OF ANTI-MADECASSOSIDE POLYCLONAL ANTIBODY AND ITS APPLICATION FOR QUALITY CONTROL OF CENTELLA ASIATICA (L.) URBAN
}

\author{
(C) PutalunWaraporn ${ }^{1,2}$, Tassanawat Patcharin ${ }^{1,2}$, Yusakul Gorawit ${ }^{1,2}$, Sritularak \\ Boonchoo $^{3}$, Juengwatanatrakul Thaweesak ${ }^{4}$, Tanaka Hiroyuki ${ }^{5}$
}

\begin{abstract}
${ }^{1}$ Faculty of Pharmaceutical Sciences, Khon Kaen University, Khon Kaen, 40002, Thailand. ${ }^{2}$ Research Group for Pharmaceutical Activities of Natural Products using Pharmaceutical Biotechnology (PANPB), National Research UniversityKhon Kaen University, Khon Kaen 40002, Thailand. ${ }^{3}$ Faculty of Pharmaceutical Sciences, Chulalongkorn University, Bangkok 10330, Thailand. ${ }^{4}$ Faculty of Pharmaceutical Sciences, Ubon Ratchathani University, Thailand.

${ }^{5}$ Graduate School of Pharmaceutical Sciences, Kyushu University, Fukuoka 812-8582, Japan
\end{abstract}

Madecassoside is the major triterpenoid saponins derived from Centella asiatica (L.) Urban, a medicinal plant used in Ayurvedic medicine for centuries. Madecassoside possesses various biological activities, including antidepressant effect (1), burn wound healing and anti-inflammatory (2). In this study, we developed the immunoassay method for quantitative analysis of the major compounds in C. asiatica. A sensitive indirect competitive enzyme-linked immunosorbent assay (ELISA) was developed using polyclonal antibody against madecassoside. Madecassoside-bovine serum albumin conjugate was immunized to rabbits for producing polyclonal antibody. The results showed that the antibodies were specific for madecassoside and aisaticoside. The range of the assay extends from 0.39 to $50 \mu \mathrm{g} / \mathrm{ml}$. A good correlation between ELISA and HPLC methods was obtained when analysis of total triterpenoid saponins in the crude extracts of plant samples. In addition, the products containing $C$. asiatica in various preparations were determined triterpenoid saponins content by competitive ELISA. The developed ELISA method can be used for quality assessment of $C$. asiatica and their products. References: (1) Liu, M. R., Han, T., Chen, Y., Qin, L. P., Zheng, H.C. and Rui, Y.C. (2004). Journal of Chinese Integrative Medicine, 2 (6), 440-444. (2) Li, H., Gong, X., Zhang, L., Zhang, Z., Luo, F., and Zhou, Q. (2009). Phytomedicine, 16 (6-7), 538-546.

\section{METABOLIC EFFECTS OF CITRUS GRANDIS WHOLE FRUITS EXTRACT IN THE STREPTOZOTOCIN-INDUCED DIABETIC RATS}

\section{(C) Raasmaja Atso ${ }^{1}$, Kovaleva Maria A. ${ }^{2}$, Makarova Marina N. ${ }^{2}$, Li Xiang Ming ${ }^{3}$, Zou Jianqiang ${ }^{3}$, Zhu Guo-Guang ${ }^{3}$, Pozharitskaya Olga N. ${ }^{2}$, Makarov Valery G. ${ }^{2}$, Shikov Alexander N. ${ }^{2}$, Hiltunen Raimo ${ }^{4}$}

${ }^{1}$ Division of Pharmacology \& Toxicology, Faculty of Pharmacy, University of Helsinki, P. O. Box 56 (Viikinkaari 5E), FIN-00014 Finland. ${ }^{2}$ St-Petersburg Institute of Pharmacy, 47/5, Piskarevsky pr., 195067, St.-Petersburg, Russia.

${ }^{3}$ Guangdong Huazhou Green Life Co., Ltd., Huazhou City, Guangdong, PRC. ${ }^{4}$ Division of Pharmaceutical Biology, Faculty of Pharmacy, University of Helsinki, P. O. Box 56 (Viikinkaari 5E), FIN-00014 Finland

Citrus grandis fruits are used as a folk remedy in Korea for hangovers, peel of the fruit in Taiwan for the treatment of stomach ache, in Cameroon traditional medicine for treatment of hypertension, in Chinese medicine as antitussive, expectorant and antiasthmatic. Dried Citrus peels, known as Chenpi are used in 